\title{
Pérdida de resincronización biventricular. Causa inhabitual. Caso clínico
}

\author{
Ismael Vergara S, Patricia Frangini S, Rolando G onzález A, \\ Damián Alonso Ma.
}

Loss of biventricular resynchronization due to an uncommon cause. Report of one case

Cardiac resynchronization therapy is a non-pharmacological treatment for patients with dilated cardiomyophaty and congestive heart failure. The success of this therapy depends of permanent biventricular stimulation. We report an 84 year-old man, with intermittent loss of biventricular pacemaker stimulation despite having adequate sensing and stimulation thresholds in the right atrium and both ventricles. The problem was solved after correcting some programming parameters (Rev Méd Chile 2006; 134: 887-92).

(Key words: Cardiac pacemaker, artificial; Cardiomyopathies; Cardiomyopathy, dilated; Heart block)

Recibido el 1 de septiembre, 2005. Aceptado el 17 de noviembre, 2005.

Laboratorio de Electrofisiología Cardíaca, Departamento de Enfermedades Cardiovasculares, Facultad de Medicina, Pontificia Universidad Católica de Chile. Santiago de Chile.

aTécnico en estimulación cardíaca

L a terapia de estimulación con resincronización biventricular en pacientes con miocardiopatía dilatada e insuficiencia cardíaca congestiva avanzada, ha sido validada y aceptada, debido a que produce mejoría en la calidad de vida, función ventricular y en la sobrevida ${ }^{1-6}$. Para lograr esta resincronización, se requiere obtener una adecuada sincronía aurículo-ventricular e interventricular, manteniendo $100 \%$ de estimulación biventricular. Sin embargo, debido a que los dispositivos utiliza-

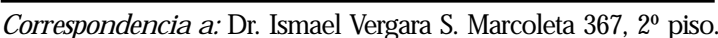
Teléfono: 3546402. Fax: 6327306. E mail: ivergara@med.puc.cl dos son cada día más complejos y con más funciones programables, no basta obtener parámetros adecuados de funcionamiento de los electrodos implantados, para asegurar el funcionamiento óptimo del dispositivo.

Presentamos el caso clínico de un paciente con miocardiopatía dilatada, enfermedad del nódulo sinusal y bloqueo aurículo-ventricular de primer grado, sometido a terapia de resincronización biventricular, en quien, pese a constatarse adecuado funcionamiento de los electrodos y programación correcta del intervalo aurículo-ventricular, se observó estimulación biventricular intermitente. 


\section{CASO CĹNIICO}

Hombre de 84 años, autovalente, portador de insuficiencia renal crónica etapa III y miocardiopatía dilatada idiopática en capacidad funcional III, con bloqueo aurículo-ventricular de primer grado (intervalo PR de 260-280 ms), y bloqueo completo de rama derecha (QRS de $160 \mathrm{~ms}$ de duración). En control ambulatorio se solicitó Holter de arritmias, que demostró enfermedad del nódulo sinusal y extrasistolía ventricular monomorfa aislada frecuente. Se le recomendó implante de marcapaso definitivo con resincronización biventricular, previo al inicio de tratamiento antiarrítmico. Se implantó sin incidentes un marcapaso Medtronic ${ }^{\circledR}$ Insync III modelo 8042, con electrodos endocavitarios bipolares Medtronic ${ }^{\circledR}$, modelo 4076, en la aurícula y ventrículo derecho, y electrodo bipolar de seno coronario Medtronic ${ }^{\circledR}$ modelo 4194, logrando parámetros adecuados de funcionamiento de todos los electrodos en el implante. El dispositivo quedó programado con frecuencia de estimulación entre 60 y 120 latidos por min, con intervalo aurículo-ventricular sensado de $130 \mathrm{~ms}$ y estimulado de $110 \mathrm{~ms}$, con output de estimulación de 4,0 Volt y 0,4 ms en aurícula derecha y ventrículo derecho y de 5,0 Volt y 0,4 ms en ventrículo izquierdo, con periodo refractario auricular post-ventricular (PVARP) en valor nominal y la función de prevención de taquicardia mediada por marcapaso activa.

El electrocardiograma de control, tomado a las $24 \mathrm{~h}$ post-implante, mostró ritmo sinusal con conducción aurículo-ventricular espontánea, con bloqueo aurículo-ventricular de primer grado y bloqueo completo de rama derecha, sin espigas de estimulación (Figura 1). La radiografía de tórax mostró los electrodos en posición adecua$\mathrm{da}$, sin evidencias de desplazamiento. La interrogación del dispositivo, mediante telemetría, mostró funcionamiento adecuado de los electrodos. Se midió en la aurícula una onda $\mathrm{P}$ sensada de 1,0-1,4 mV, umbral de estimulación $<0,5 \mathrm{~V}$ a $0,4 \mathrm{~ms}$, con impedancia de estimulación de 355 Ohms; en el ventrículo derecho se midió onda $\mathrm{R}$ sensada de 15,68-22,4 mV, umbral de estimulación $<0,5 \mathrm{~V}$ a $0,4 \mathrm{~ms}$, con impedancia de estimulación de $485 \mathrm{Ohms;}$ y en el ventrículo izquierdo, se midió onda $\mathrm{R}$ sensada de 4,0-5,6 $\mathrm{mV}$, umbral de estimulación de $1,0 \mathrm{~V}$ a $0,4 \mathrm{~ms}$, con impedancia de estimulación de 552 Ohms. Los histogramas mostraron sólo 94\% de estimulación biventricular desde el implante. Durante la interrogación, se observó que los episodios de

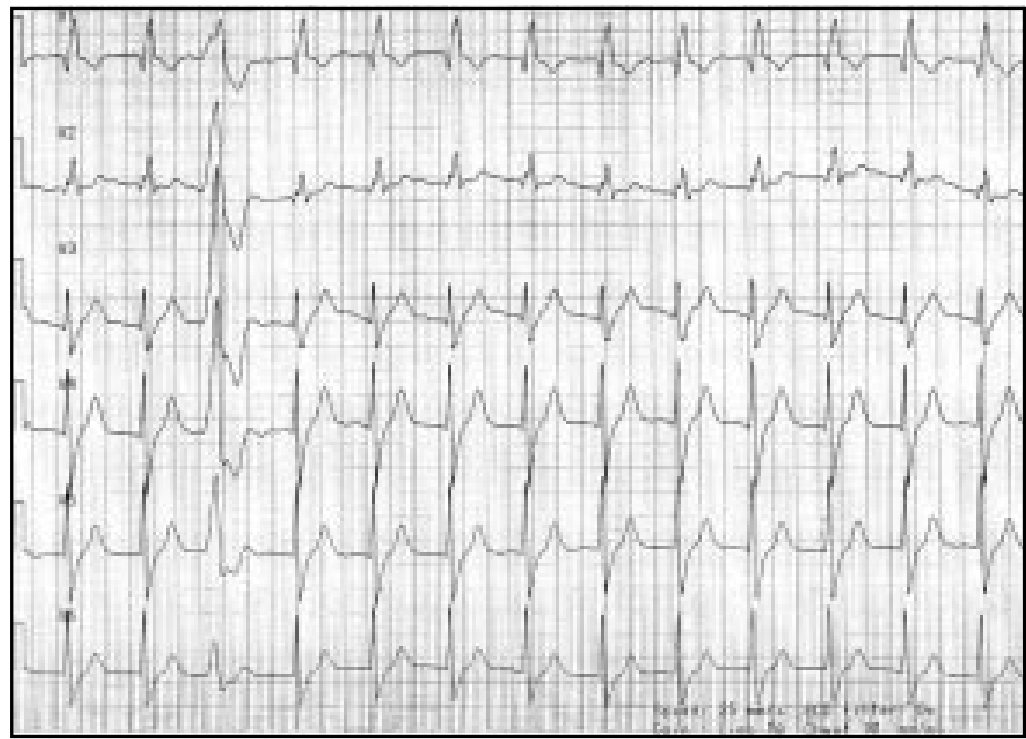

Figura 1. Electrocardiograma de superficie $24 \mathrm{~h}$ postimplante (derivaciones precordiales). Se observa ausencia de espigas de estimulación. El ritmo es sinusal con frecuencia de 95 latidos por min, conducido 1:1 con PR de $260 \mathrm{~ms}$, con imagen de bloqueo completo de rama derecha y extrasistolia ventricular aislada. 
conducción aurículo-ventricular espontánea se producían siempre después de un extrasístole ventricular, el cual era adecuadamente sensado y seguido de una onda P sinusal (actividad auricular espontánea) sensada dentro del período refractario auricular post-ventricular (PVARP). Al existir conducción aurículo-ventricular, el complejo QRS provocado siguiente era sensado antes de completarse el intervalo de escape auricular, volviendo a repetirse este fenómeno completo con el siguiente latido, perdiéndose»la estimulación biventricular (Figura 2). Se optó por reprogramar el PVARP desde su valor nominal dinámico a un valor fijo de $310 \mathrm{~ms}$, con lo que no se volvió a observar el fenómeno descrito. Sin embargo, el ECG de control del día siguiente, nuevamente mostró ausencia de estimulación ventricular. La reinterrogación del dispositivo mostró funcionamiento adecuado de los electrodos ventriculares, y se observó que en forma intermitente ocurría falla de sensado auricular manteniendo un buen umbral de estimulación. La onda $\mathrm{P}$ sensada disminuyó a un valor de $0,5-0,7$ $\mathrm{mV}$, sin evidencias radiológicas de desplazamiento. La ausencia de sensado auricular se asociaba a sensado del QRS espontáneo a una frecuencia por sobre la frecuencia mínima programada, por lo que había inhibición de la estimulación del marcapaso (Figura 3). Esto se corrigió programando la sensibilidad auricular a su valor máximo de 0,18 mV. El seguimiento clínico y Holter de control de electrocardiograma posterior, han mostrado evolución favorable y estimulación biventricular 100\% efectiva. En control ambulatorio se optó por desactivar la función de prevención de taquicardia mediada por marcapaso.

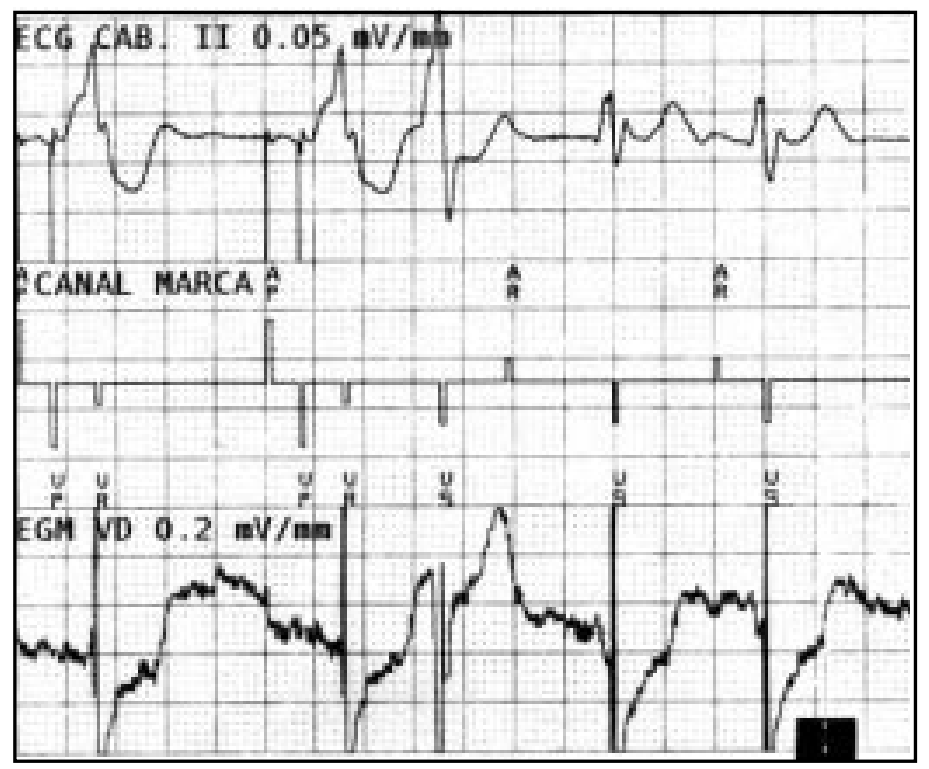

Figura 2. Registro de la interrogación del dispositivo (velocidad del papel $25 \mathrm{~mm} / \mathrm{s}$ ). El primer canal corresponde a electrocardiograma de superficie (derivación DII), el segundo canal muestra los marcadores de eventos registrados por el dispositivo, y el tercer canal corresponde al electrograma del ventrículo derecho. Los primeros dos complejos corresponden a estimulación auricular y ventricular, el tercer complejo es un extrasístole ventricular adecuadamente sensado, apareciendo la onda P sinusal siguiente con un intervalo VA de 230 ms, dentro del periodo refractario auricular post-ventricular, marcada como AR. El cuarto complejo QRS corresponde a conducción espontánea del ritmo sinusal. El siguiente complejo P-QRS muestra ausencia de estimulación bicameral, cayendo la aurícula nuevamente en periodo refractario.

Abreviaciones. AP: evento auncular estimulado. AR: evento aunicular sensado dentro del periodo refractario aunicular. AS: evento auricular sensado fuera del periodo refractario. VP: evento ventricular sensado. VR: evento ventricular sensado dentro del periodo refractario ventricular. VS: evento ventricular sensado fuera del periodo refractario ventricular. 


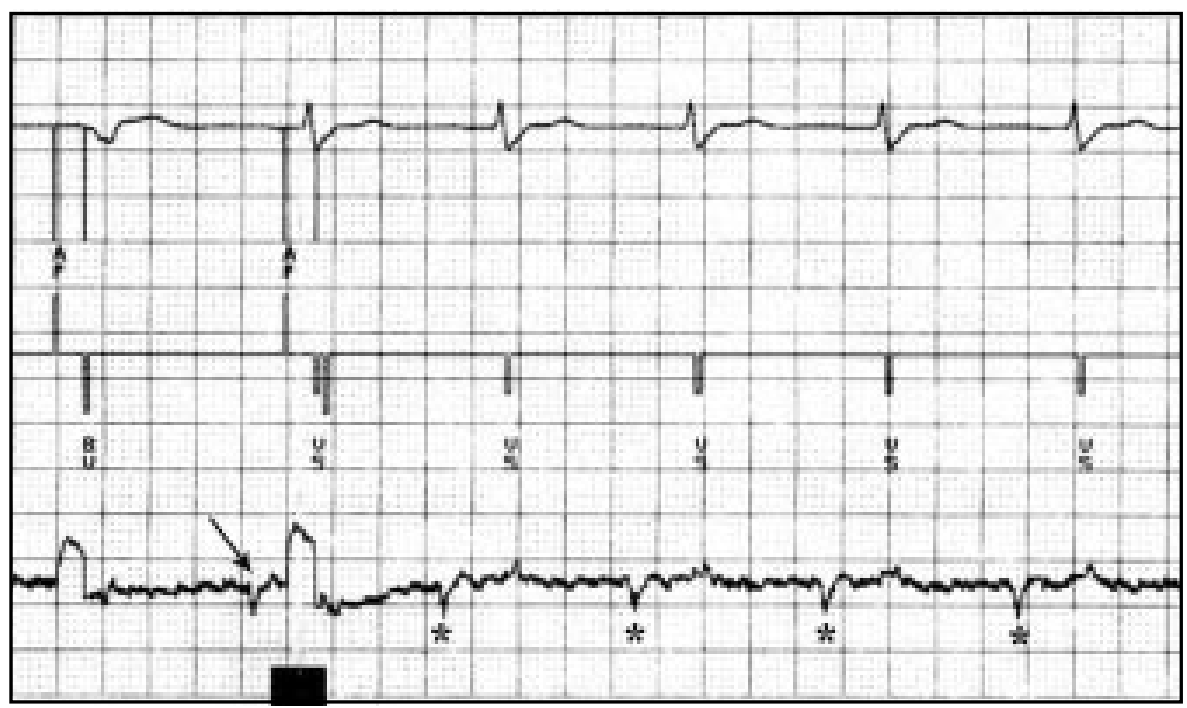

Figura 3. Registro de la interrogación del dispositivo (velocidad del papel $25 \mathrm{~mm} / \mathrm{s}$ ). El primer canal corresponde a electrocardiograma de superficie (derivación DI), el segundo canal muestra los marcadores de eventos registrados por el dispositivo, y el tercer canal corresponde al electrograma de la aunícula derecha. El primer complejo corresponde a estimulación auricular y ventricular. En el segundo complejo se produce falla de sensado de la aunícula (flecha), seguida de espiga de estimulación auricular inefectiva y sensado del QRS producto de la onda P sinusal precedente. A partir del tercer complejo la actividad auricular no es sensada $\left(^{*}\right.$ y el marcapaso sólo detecta el electrograma ventricular con una marca VS, con ausencia de espigas de estimulación.

Abreviaciones. AP: evento auricular estimulado. BV: estimulación biventricular. VS: evento ventricular sensado fuera del periodo refractario ventricular.

\section{Discusión}

Como se mencionó previamente, varios estudios han demostrado que los pacientes sometidos a terapia de resincronización biventricular asociada a terapia farmacológica óptima, presentan una significativa mejoría de sus parámetros clínicos, regresando en al menos una clase funcional de la New York Heart Association, y rindiendo mejor en el test de caminata de seis min y en la medición de consumo máximo de $\mathrm{O}_{2}$, lo que se refleja en alivio de los síntomas, mejoría en las encuestas de calidad de vida, reducción en el número de hospitalizaciones y mejoría en las curvas de sobrevida, resultados que se mantienen a 1 y 2 años plazo ${ }^{1-8}$. Esto ha llevado a que las indicaciones de esta terapia se hayan ampliado y se le ofrezca a pacientes con miocardiopatía dilatada e insuficiencia cardíaca avanzada, con QRS ancho e imagen de bloqueo completo de rama derecha con demostración de asincronía ventricular. Si uno de estos pacientes requiere de estimulación ventricular, como es el caso del paciente presentado, probablemente la estimulación biventricular sea la mejor opción ya que se sabe que la estimulación ventricular derecha provoca deterioro significativo del débito cardíaco y asincronía ventricular iatrogénica $^{9-12}$.

Para que la terapia de resincronización biventricular sea efectiva, se necesita que exista estimulación biventricular permanente. Se ha descrito que en un seguimiento a más de 2 años, hasta $36 \%$ de los pacientes pueden presentar pérdida de la estimulación biventricular en forma permanente 0 transitoria, especialmente en relación a aparición de taquiarritmias auriculares (18\%), pérdida de captura ventricular izquierda (10\%) y falla de sensado auricular derecho (1\%) ${ }^{13-15}$. La programación inadecuada también puede llevar a pérdida del beneficio de la estimulación biventricular. En el 
caso de este paciente, al momento del implante el intervalo PVARP se dejó programado en su valor nominal, que en el marcapaso Medtronic ${ }^{\circledR}$ Insync III modelo 8042 corresponde a un valor dinámico, dependiente de la frecuencia cardíaca, mínimo de $250 \mathrm{~ms}$ y máximo de $400 \mathrm{~ms}$. Al ocurrir un extrasístole ventricular, el algoritmo para prevenir taquicardia mediada por marcapaso prolonga automáticamente el PVARP, para evitar que la probable onda $\mathrm{P}$ retrógrada sea sensada y seguida de estimulación ventricular. Debido a que la frecuencia auricular espontánea del paciente en reposo era de alrededor de 90 latidos por minuto, la extensión del PVARP post extrasístole ventricular producía que la siguiente onda $\mathrm{P}$ sinusal fuera sensada dentro del período refractario y, por lo tanto, no gatillase estimulación biventricular. Al haber conducción espontánea, el complejo QRS siguiente es sensado por el electrodo ventricular antes de completar el ciclo de la frecuencia mínima de estimulación, perdiéndose la estimulación biventricular. Finalmente, al disminuir espontáneamente la frecuencia sinusal, esta actividad auricular era sensada fuera del PVARP y al ser reconocida como actividad, gatillaba la estimulación biventricular en el latido siguiente, respetando el intervalo AV programado. El problema fue solucionado programando el PVARP a un valor fijo de $310 \mathrm{~ms}$. Hay dos casos descritos en que este fenómeno fue gatillado por el algoritmo de prevención de taquicardia mediada por marcapaso sin ocurrencia de un extrasístole ventricular ${ }^{16,17}$. El marcapaso Medtro-

\section{REFERENCIAS}

1. Cieland J, Daubert JC, Erdmann E, Freemantle N, Gras D, Kappenberger L et al. The effect of cardiac resynchronization on morbidity and mortality in heart failure. N Engl J Med 2005; 352: 1539-49.

2. Cazeau S, Leclerco C, Lavergne T, Walter S, Varma C, LiNDe C ET AL, FOR MUSTIC Study Investigators. Effects of multisite biventricular pacing in patients with heart failure and intraventricular conduction delay. N Engl J Med 2001; 344: 873-80.

3. Abraham WT, Fisher WG, Smith AL, Delurgio DB, LeON AR, Loh E et al, For the MIRACLE Study nic ${ }^{\circledR}$ Insync III detecta taquicardia mediada por marcapaso después de ocho latidos consecutivos que cumplan los tres siguientes criterios: (a) intervalo VA $<400 \mathrm{~ms}$, (b) el intervalo se inicia con un evento ventricular estimulado, (c) el intervalo termina con un evento auricular sensado fuera del periodo refractario ${ }^{18}$. Esto, asociado a bloqueo aurículo-ventricular de primer grado, favorece la pérdida de la estimulación biventricular porque el próximo latido auricular sigue cayendo dentro del intervalo PVARP. En estas circunstancias se recomienda programar la función de taquicardia mediada por marcapaso en modo desactivado.

La falla de sensado auricular ha sido descrita en $1 \%$ de los pacientes sometidos a terapia de resincronización biventricular. En la serie de 512 pacientes de Knight et al, en los 5 pacientes con falla de sensado auricular, todos los casos fueron solucionados con reprogramación o revisión quirúrgica del electrodo ${ }^{13}$. En nuestro paciente, la reprogramación fue suficiente para solucionar este problema, dado que este dispositivo permite un margen de programación de la sensibilidad auricular entre $0,18 \mathrm{mV}$ y $4,0 \mathrm{mV}$.

La ocurrencia de falla intermitente de estimulación biventricular puede afectar significativamente la respuesta del paciente a la terapia, y es necesario evaluar un amplio espectro de posibilidades diagnósticas para lograr su corrección. Esta es una de las razones por lo que la terapia de resincronización biventricular debe ser manejada por médicos especialistas en marcapasos.

GrouP. Cardiac resynchronization in chronic heart failure. N Eng J Med 2002; 346: 1845-53.

4. Bradiey JB, Bradiey EA, Baughman KL, Berger RD, Calkins H, Goodman SN et al. Cardiac resynchronization and death from progressive heart failure. A meta-analysis of randomized controlled studies. JAMA 2003; 289: 730-40.

5. Young JB, Abraham WT, Smith AL, Leon AR, LEBERMAN R, WILKOFF B ET AL, FOR THE MIRACLE ICD Trial InVESTIGators. Combined cardiac resynchronization and implantable cardioversion defibrillation in advanced chronic heart failure. The MIRACLE ICD trial. JAMA 2003; 289: 2685-94. 
6. Bristow MR, Saxon LA, Boehmer J, Krueger S, Kass DA, DeMarco T ET AL, FOR THE COMPANION INVESTIGATORS. Cardiac-resynchronization therapy with or without an implantable defibrillator in advanced chronic heart failure. N Engl J Med 2004; 350: 2140-50.

7. Linde C, Lecierco C, ReX S, Garrigue S, Lavergne T, Cazeau S et al, (MUSTIC Study Group). Long-term benefits of biventricular pacing in congestive heart failure: Results from the Multisite Stimulation in Cardiomyopathy (MUSTIC) Study. J Am Coll Cardiol 2002; 40: 111-18.

8. Linde C, Braunschweig F, Gadier F, Baileul C, DAUBERT JC. Long-term improvement in quality of life by biventricular pacing in patients with chronic heart failure: Results from the MUSTIC study. Am J Cardiol 203; 91: 1090-95.

9. Montagna R, Asenjo $R$, Morris R, Schumacher E, Aguayo R, Ortiz M et al. Ventricular resynchronization therapy in patients with previous pacemaker for the management of advanced refractory heart failure. Rev Chil Cardiol 2005; 24: 48-59.

10. Owen CH, Esposito DJ, Davis JW, Glower DD. The effects of ventricular pacing on left ventricular geometry, function, myocardial oxygen consumption, and efficiency of contraction in conscious dogs. Pacing Clin Electrophysiol 1998; 21: 1417-29.
11. Nielsen J, Kristensen L, Andersen H, Mortensen P, Pedersen O, Pedersen A. A randomized comparison of atrial and dual-chamber pacing in 177 consecutive patients with sick sinus syndrome. J Am Coll Cardiol 2003; 42: 614-23.

12. Giudici MC, Thornburg GA, Buck DL, Coyne EP, Walton MC, Paul DL et al. Comparison of right ventricular outflow tract and apical lead permanent pacing on cardiac output. Am J Cardiol 1997; 79: 209-12.

13. Knight BP, Desai A, Coman J, Faddis M, Yong P. Long-term retention of cardiac resynchronization therapy. J Am Coll Cardiol 2004; 44: 72-7.

14. Van Erven L, Molhoek SG, Van der Wall EE, Schalu MJ. Cyclic appropriate mode switching and inappropriate back switching of a biventricular pacemaker during atrial tachyarrhythmia. Pacing Clin Electrophysiol 2004; 27: 249-51.

15. Eidelman RS, Dieguez FJ, Tolentino A, Zebede J. Malfunction of a biventricular pacing device? Pacing Clin Electrophysiol 2004; 27: 1453-4.

16. Richardson K, Cook K, Wang PJ, Al-Ahmad Amin. Loss of biventricular pacing: What is the mechanism? Heart Rhythm 2005; 2: 110-1.

17. BAROLD SS, Herweg B. Mysterious loss of resynchronization during biventricular pacing. Pacing Clin Electrophysiol 2005; 28: 571-2.

18. Medtronic Inc. Insync III device model 8042 Reference Manual 2000; 5.5-5.7. 